\title{
One-dimensional Cooper pairing
}

\author{
R. Mendoza ${ }^{\mathrm{a}}$, M. Fortes ${ }^{\mathrm{a}, *}$, M. de Llano ${ }^{\mathrm{b}}$, M.A. Solís ${ }^{\mathrm{a}}$ \\ ${ }^{a}$ Instituto de Física, UNAM, Apdo. Postal 20-364, 01000 México D.F., MEXICO \\ ${ }^{b}$ Instituto de Investigaciones en Materiales, UNAM, Apdo. Postal 70-360, 04510 México D.F., MEXICO
}

\begin{abstract}
We study electron pairing in a one-dimensional (1D) fermion gas at zero temperature under zero- and finite-range, attractive, two-body interactions. The binding energy of Cooper pairs (CPs) with zero total or center-of-mass momentum (CMM) increases with attraction strength and decreases with interaction range for fixed strength. The excitation energy of 1D CPs with nonzero CMM display novel, unique properties. It satisfies a dispersion relation with two branches: a phonon-like linear excitation for small CP CMM; this is followed by roton-like quadratic excitation minimum for CMM greater than twice the Fermi wavenumber, but only above a minimum threshold attraction strength. The expected quadratic-in-CMM dispersion in vacuo when the Fermi wavenumber is set to zero is recovered for any coupling. This paper completes a three-part exploration initiated in 2D and continued in 3D.
\end{abstract}

Keywords: Cooper pairing, BCS, roton mode, linear mode

\section{Introduction}

Now just over a half-century old, the 1957 Bardeen, Cooper and Schrieffer (BCS) theory of superconductivity [1] is rightly regarded as one of the most striking achievements of theoretical many-body physics. It has been ranked along with the band theory of solids and the Landau theory of Fermi liquids, both single-particle formalisms. The central concept of the BCS theory is that of fermion pairings. In the original model of Cooper [2] they were simply two-electron bound states relative to a full Fermi sea of the many-electron system. In the BCS theory this original concept was incorporated into a many-body ground-state variational trial wavefunction in which all electrons share "pairing correlations." The theory, though valid only for weak-coupling, not only provided a microscopic model for superconductivity, but it also made many highly specific and quantitative predictions including explaining the isotope effect, predicting the $T=0$ energy gap $\Delta(0)$ obeying the universal relation, $2 \Delta(0) \simeq 3.53 k_{B} T_{c}$ where the transition temperature $T_{c}$ is the smallest solution of $\Delta\left(T_{c}\right)=0$, and in explaining $T$-dependences of ultrasonic attenuation and nuclear magnetic resonance relaxation rates [3]. Stronger coupling would not guarantee higher-than-pair clusterings, e.g., tetramers, etc., since these higher-order charge clusters have not been detected at all in magnetic-flux quantization measurements in either conventional superconductors (specifically tin [4] and lead [5]) nor in so-called "high- $T_{c}$ " compounds such as $\mathrm{YBaCuO}$ [6]: the smallest flux unit observed is $h c / 2 e$ rather than the originally expected $h c / e$ and it is never smaller than $h c / 2 e$.

For many decades the BCS theory, including its extensions into the strong-coupling regime, appeared to be capable of explaining all of the then known superconducting elements and compounds. This situation continued while the highest $T_{c}$ value for any superconductor (SC) was $23 \mathrm{~K}$, until the discovery [7] in 1986 of the first high- $T_{c}$ cuprate SC $\mathrm{La}_{2-x} \mathrm{Ba}_{x} \mathrm{CuO}_{4}$ having a $T_{c} \simeq 35 \mathrm{~K}$. The discovery [8] of superconductivity at $92 \mathrm{~K}$ in $\mathrm{YBa}_{2} \mathrm{Cu}_{3} \mathrm{O}_{7-\delta}$ was followed by a search for materials with even higher $T_{c} \mathrm{~s}$ and lead, within just seven years to the highest- $T_{c}$ superconductor known and fully confirmed to date, the $\mathrm{HgBaCaCuO}$ cuprate [9] with a $T_{c} \simeq 164 \mathrm{~K}$ under very high pressure $(\simeq 310,000$ atm).

\footnotetext{
${ }^{*}$ Corresponding author

Email addresses: ramen@fisica.unam.mx (R. Mendoza), fortes@fisica.unam.mx (M. Fortes), dellano@servidor.unam.mx (M. de Llano), masolis@fisica.unam.mx (M.A. Solís)
} 
Almost a quarter century after the discovery of high-temperature SCs in cuprate materials, it is clear that many important questions still remain to be answered. As well as the still unresolved problem of the pairing dynamical mechanism and many-body excitations in the normal state of the high $T_{c}$ cuprate materials, there are now also many other recently discovered materials where it is unlikely that BCS theory is applicable, at least in its original form. These include oxide materials (such as the cubic bismuthate $\mathrm{Ba}_{1-x} \mathrm{~K}_{x} \mathrm{BiO}_{3}$ ), borides (such as $\mathrm{MgB}_{2}$ ), borocarbides (e.g. $\mathrm{YNi}_{2} \mathrm{~B}_{2} \mathrm{C}$ ), carbon-based materials (including fullerides, nanotubes, intercalated graphite, and organic conductors), and new high pressure phases of elements [10] (such as Fe, S and Ca) and simple binary and ternary compounds. All of these classes of materials have shown superconductivity above $10 \mathrm{~K}$, including several up to nearly $40 \mathrm{~K}$. Superconductivity at up to $84 \mathrm{~K}$ has even been reported in a cubic ruthenate [11, 12, 13].

The ongoing debate about the pairing dynamical mechanism in cuprate high $T_{c}$ materials has broadly led to two main schools of thought. On the one hand P.W. Anderson argued from the very beginning [14] that cuprate materials are in a completely different class from other superconducting materials, and as such they must have a completely new pairing mechanism quite different from the BCS theory. In addition to his original "resonating-valence-bond" (RVB) model a large range of theories have focussed on superconductivity driven chiefly by repulsive interactions dominated by the on-site Coulomb-repulsion Hubbard $U$. These include gauge theories [15], spin-fluctuation theories [16, 17, 18], and the "Gossammer superconductivity" picture of Laughlin [19]. The discovery of a $d_{x^{2}-y^{2}}$ symmetry order parameter [20, 21] is generally consistent with pairing mechanisms deriving from a large positive $U$, and there is some numerical evidence for a $d_{x^{2}-y^{2}}$ symmetry ground state in the two-dimensional square lattice Hubbard model 22]. However, it remains unclear whether the positive $U$ Hubbard model alone can describe the hugely complex normal and superconducting state phenomenology of the cuprate materials [23] including the characteristic doping dependences, pseudogaps, marginal Fermi liquid normal state, isotope effects, and lattice inhomogeneities such as stripes.

On the other hand, many others have taken the view that it is not a completely new theory that is needed, but rather that the BCS theory should be extended and/or generalized to describe these new materials. This approach has the advantage of building upon the foundations of BCS, and furthermore does not necessarily imply that cuprate superconductivity is in a completely new class of SCs. Rather, they may be related to other materials but just in a new parameter regime where the usual approximations of BCS (even including Eliashberg strong-coupling corrections) may not be adequate. Some of the many theoretical models which have been examined in this context include: boson-fermion models [24, 25, 26, 27, 28, 29], bipolarons [30], the "pre-formed pair" or BCS-BEC crossover scenario 31, 32, 33, 34, 35], non-adiabatic superconductivity [36], and generalized Bose-Einstein condensation of Cooper pairs 37, 38, 39].

In this work we study electron pairing in a 1D Fermi gas under zero- and finite-range, two-fermion interactions to address some novel and unique properties of CPs in 1D as compared with the 2D [40] and 3D [41] cases. In Section 2 we review the Cooper pairing mechanism in a simplified model where two particles near a static Fermi level interact at $T=0$. In Section 3 we obtain exact solutions for different types of separable interactions. The properties of the bound pairs are discussed in Section 4 where we derive the dispersion relation for arbitrary values of the center-of-mass momenta $(\mathrm{CMM}) \hbar K$. Section 5 presents results for pairs of particles interacting in a more general case where a range parameter is introduced. Section 6 discusses our conclusions.

\section{Cooper pairing}

To define the original Cooper-pair (CP) problem [2] consider a system of $N$ identical fermions in $d$-dimensions interacting through an attractive two-body potential to study the effects of pairing at nonzero total or center-of-mass momenta under different types of interaction. At zero temperature, we assume that the background $(N-2)$-particle system is in the ground state of an ideal Fermi gas with interactions occurring only in the vicinity of the Fermi level. The Schrödinger equation for two particles in momentum space is

$$
\left(\mathbf{p}_{1}^{2} / 2 m+\mathbf{p}_{2}^{2} / 2 m-E\right) \Phi\left(\mathbf{p}_{1}, \mathbf{p}_{2}\right)+\sum_{\mathbf{p}_{1}^{\prime} \in \mathcal{R}} \sum_{\mathbf{p}_{2}^{\prime} \in \mathcal{R}} V\left(\mathbf{p}_{1}, \mathbf{p}_{2} ; \mathbf{p}_{1}^{\prime}, \mathbf{p}_{2}^{\prime}\right) \Phi\left(\mathbf{p}_{1}^{\prime}, \mathbf{p}_{2}^{\prime}\right)=0
$$


where $\mathcal{R}$ denotes the region of available states above the Fermi level and $V$ is the two-body interaction. Introducing the CMM $\mathbf{P}$ and relative momentum $\mathbf{p}$ as

$$
\mathbf{P} \equiv \hbar \mathbf{K}=\hbar \mathbf{k}_{1}+\hbar \mathbf{k}_{2} \quad \text { and } \quad \mathbf{p} \equiv \hbar \mathbf{k}=\frac{1}{2} \hbar\left(\mathbf{k}_{1}-\mathbf{k}_{2}\right)
$$

the equation of motion for two particles above the Fermi level becomes

$$
\left[\hbar^{2} \mathbf{k}^{2} / m+\hbar^{2} \mathbf{K}^{2} / 4 m-E_{K}\right] \Phi(\mathbf{k}, \mathbf{K})+\sum_{k^{\prime}>k_{F}} V_{\mathbf{k}, \mathbf{k}^{\prime}}^{\mathbf{K}} \Phi\left(\mathbf{k}^{\prime}, \mathbf{K}\right)=0,
$$

where $\Phi(\mathbf{k}, \mathbf{K})$ is the two-particle wavefunction. When the interaction $V_{\mathbf{k}, \mathbf{k}^{\prime}}^{\mathbf{K}} \equiv V\left(\mathbf{K} / 2+\mathbf{k}, \mathbf{K} / 2-\mathbf{k} ; \mathbf{K} / 2+\mathbf{k}^{\prime}, \mathbf{K} / 2-\mathbf{k}^{\prime}\right)$ commutes with the CMM operator associated with $\hbar \mathbf{K}, \Phi(\mathbf{k}, \mathbf{K})=\varphi(\mathbf{k}) \Psi(\mathbf{K})$ and the center-of mass wavefunction $\Psi(\mathbf{K})$ can be factored out. In $3 \mathrm{D} V_{\mathbf{k}, \mathbf{k}^{\prime}}^{\mathbf{K}}$ can be expanded in partial waves as in the case of the Cooper model interaction where already the $l=0$ contribution leads to a correlated ground-state with paired particles at the Fermi surface providing the crucial ingredient in the formulation of the BCS theory [1] of superconductivity.

Here we focus on some novel and unique properties of CPs in 1D, as compared with the 2D [40] and 3D [41] cases. We start from a nonlocal, separable interaction given by

$$
V_{\mathbf{k}, \mathbf{k}^{\prime}}^{\mathbf{K}}=-V_{0} g(k) g\left(k^{\prime}\right)
$$

with $V_{0}>0$. This includes all cases of physical interest such as the Cooper 2], the BCS [1], the zero-range or contact [40, 41, 42, 43] model interactions, as well as the finite-range model interactions as introduced by Nozières and SchmittRink [44] that have been used in the description of superconductors, superfluids, Bose-Einstein condensates, as well as the BCS-BEC crossover [31, 32, 33, 34, 35] picture. The separable form (44) assumes that such an expression is valid in each partial-wave channel and may vary for angular momentum states different from zero in $2 \mathrm{D}$ and $3 \mathrm{D}$. In all these cases, (3) has an analytical solution given by

$$
\varphi(k)=\frac{V_{0} g(k) A}{\hbar^{2} k^{2} / m+\hbar^{2} K^{2} / 4 m-E_{K}}
$$

where

$$
A \equiv \sum_{k^{\prime}>k_{F}} g\left(k^{\prime}\right) \varphi\left(k^{\prime}\right)
$$

is a constant. The combination of these two equations provides a consistency condition that is equivalent to an eigenvalue equation for $E_{K}$, namely

$$
1=V_{0} \sum_{k>k_{F}} \frac{g^{2}(k)}{\hbar^{2} k^{2} / m+\hbar^{2} K^{2} / 4 m-E_{K}} .
$$

Defining $-\Delta_{K} \equiv E_{K}-2 E_{F}$ as the pair energy with respect to twice the Fermi energy $E_{F} \equiv \hbar^{2} k_{F}^{2} / m$, the continuous limit for large $N$ and large volume is

$$
1=V_{0}(2 \pi)^{-d} L^{d} \int \frac{d^{d} k g^{2}(k)}{\hbar^{2}\left(k^{2}-k_{F}^{2}\right) / m+\Delta_{K}+\hbar^{2} K^{2} / 4 m}
$$

where $L^{d}$ is the "volume" of the $d$ dimensional system.

When the interaction occurs only in the vicinity of the Fermi energy $E_{F} \equiv \hbar^{2} k_{F}^{2} / 2 m$, and since the integrand in (17) is peaked at $k \sim k_{F}$, the integral can be reexpressed in terms of the density of states (DOS) for one spin at the Fermi level, namely

$$
\varrho_{d}\left(E_{F}\right)=m\left(\frac{L}{2 \pi \hbar}\right)^{d} c_{d}\left(2 m E_{F}\right)^{d / 2-1}
$$

where $c_{d}=4 \pi, 2 \pi, 2$ for $d=3,2,1$, respectively. 
In $1 \mathrm{D}$

$$
\varrho_{1}\left(E_{F}\right)=\frac{m L}{\pi \hbar^{2} k_{F}} .
$$

If $g(k)$ is constant around the Fermi level the usual Cooper binding-energy result can be obtained for any CMM wavenumber $K \geqslant 0$. However, this approximation is unnecessary since one can analytically integrate (7) for most interaction models of physical interest. For brevity, one can express all energies in terms of the Fermi energy and all wavenumbers in units of the Fermi wavenumber $k_{F}$, namely through dimensionless $\tilde{K}=K / k_{F}, \tilde{k}=k / k_{F}$ and $\tilde{\Delta}_{K}=\tilde{\Delta}_{K} / E_{F}$. Then (7) becomes

$$
1=\frac{\lambda}{2} \int \frac{d \tilde{k} g^{2}(\tilde{k})}{\tilde{k}^{2}+\tilde{\Delta}_{K} / 2+\tilde{K}^{2} / 4-1}
$$

where the dimensionless coupling constant is

$$
\lambda \equiv \varrho_{1}\left(E_{F}\right) V_{0}=\frac{V_{0} m L}{\pi \hbar^{2} k_{F}} .
$$

Condition (10) is the effective characteristic equation for the bound-pair energy $\Delta_{K}$ which can be solved exactly for any form factor $g(k)$ of physical interest.

\section{Cooper pairing in one dimension}

Fermi systems in 1D reveal a novel multiphase pairing mechanism that is absent in higher-dimensional systems. When pair interactions act only in the vicinity of the Fermi energy as in the case of the Cooper [2] and the BCS 1] model interactions, the available phase space has at least two discontinuous intervals for the total or CMM $\hbar K$, see Fig. 1. When $K \ll 2 k_{F}$, particles at both ends of the "dumbbell" feel the attractive interactions because their energies are close to the Fermi energy $E_{F}$. As $K$ increases there is a region where particles no longer interact due to their large momenta, until the condition $k_{F}<K / 2<k_{F}+k_{D}$ is satisfied, where $k_{D} \equiv k_{F} \sqrt{1+\hbar \omega_{D} / E_{F}}-k_{F}$ as shown in the lower part of Fig. 1. The cutoff wavenumber $k_{D}$ is related to the maximum ionic-lattice vibrational (or Debye) frequency $\omega_{D}$ through $\hbar^{2}\left(k_{F}+k_{D}\right)^{2} / 2 m \equiv E_{F}+\hbar \omega_{D}$. Clearly, for a contact interaction $\hbar \omega_{D} / E_{F} \rightarrow \infty$ so that $k_{D} \rightarrow \infty$.

The form factors $g(k)$ of the separable pairing interaction (4) are given by

$$
g(k)= \begin{cases}\theta\left(\epsilon_{1,2}-E_{F}\right) & \text { contact interaction } \\ \theta\left(\epsilon_{1,2}-E_{F}\right) \theta\left(E_{F}+\hbar \omega_{D}-\epsilon_{1,2}\right) & \text { Cooper interaction } \\ \theta\left(\epsilon_{1,2}-\left[E_{F}-\hbar \omega_{D}\right]\right) \theta\left(\left[E_{F}+\hbar \omega_{D}\right]-\epsilon_{1,2}\right) & \text { BCS interaction }\end{cases}
$$

where

$$
\theta(x) \equiv\left\{\begin{array}{l}
1 \text { for } x>0 \\
0 \text { for } x<0
\end{array}\right.
$$

and $\epsilon_{1,2}$ are the energies of particle 1 and of particle 2 given by

$$
\epsilon_{1,2} \equiv \hbar^{2} k_{1,2}^{2} / 2 m \equiv \hbar^{2}(K / 2 \pm k)^{2} / 2 m
$$

In terms of the wavenumber, the form factors in (12) are

$$
g(\tilde{k})= \begin{cases}\theta(|\tilde{k}|-[1+\tilde{K} / 2]) & \text { contact interaction } \\ \theta(|\tilde{k}|-[1+\tilde{K} / 2]) \theta\left(\sqrt{1+\hbar \omega_{D} / E_{F}}-\tilde{K} / 2-|\tilde{k}|\right) & \text { Cooper interaction } \\ \theta\left(|\tilde{k}|-\left[\sqrt{1-\hbar \omega_{D} / E_{F}}-\tilde{K} / 2\right]\right) \theta\left(\left[\sqrt{1+\hbar \omega_{D} / E_{F}}-\tilde{K} / 2\right]-|\tilde{k}|\right) & \text { BCS interaction }\end{cases}
$$

where, for definiteness, we have assumed $\tilde{K}>0$ since the $\tilde{K}<0$ sector provides the same results. In what follows we occasionally drop the tildes. 

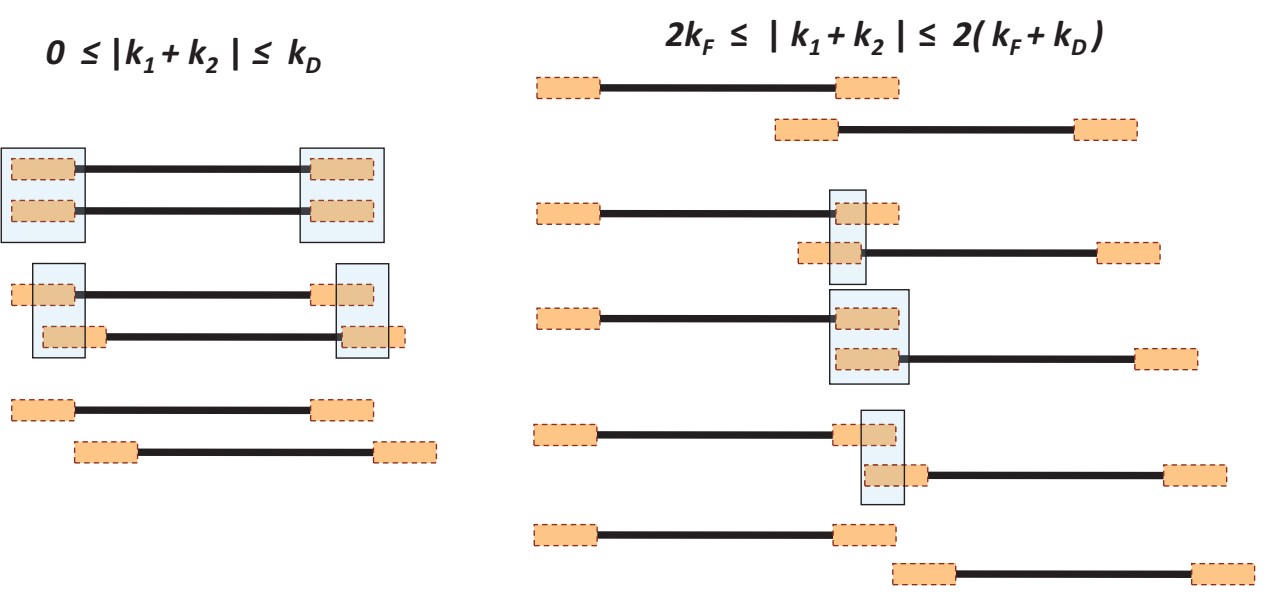

Figure 1: "Dumbbell" picture of BCS or Cooper interaction regions (shaded boxes, not in scale) in momentum space for pairs of fermions. Dumbbells are laterally separated by the total CMM $K$ of a CP. Uppermost part in the left block represents $K=0$ where the available phase-space for interactions is maximum. In contrast with higher-dimensional systems, in $1 \mathrm{D}$ there is an interval where the interaction phase space vanishes as $K$ increases from $K=k_{D}$ to $K=2 k_{F}$ (right block of diagrams).

The BCS interaction poses a problem that was first mentioned by Schrieffer (Ref. [3], p. 168) related to the singularities in (10) since the integration intervals defined in (13) may produce zeros in the denominator. However, for contact and Cooper interactions (10) can be solved exactly yielding, for $K \ll 1$,

$$
\exp \left(-2 \alpha_{K} / \lambda\right)= \begin{cases}\frac{\left(1+K / 2-\alpha_{K}\right)}{\left(1+K / 2+\alpha_{K}\right)} & \text { contact interaction } \\ \frac{\left(1+k_{D}-K / 2+\alpha_{K}\right)\left(1+K / 2-\alpha_{K}\right)}{\left(1+k_{D}-K / 2-\alpha_{K}\right)\left(1+K / 2+\alpha_{K}\right)} & \text { Cooper interaction }\end{cases}
$$

where $k_{D}$ was defined above and

$$
\alpha_{K}^{2} \equiv 1-K^{2} / 4-\Delta_{K} / 2 .
$$

For $K$ sufficiently large $\alpha_{K}^{2}$ becomes negative and the existence of stable solutions for $\Delta_{K}$ depends on the magnitude of $\lambda$. For $\lambda \ll 1$ one has, if $\beta_{K}^{2} \equiv-\alpha_{K}^{2}$,

$$
\frac{\beta_{K}}{\lambda}= \begin{cases}\pi / 2-\tan ^{-1}\left[(1+K / 2) / \beta_{K}\right] & \text { contact interaction } \\ \tan ^{-1}\left[\left(1+k_{D}-K / 2\right) / \beta_{K}\right]-\tan ^{-1}\left[(1+K / 2) / \beta_{K}\right] & \text { Cooper interaction }\end{cases}
$$

Since (14) are transcendental in the CP energy $\Delta_{K}$ (not to be confused with the BCS energy gap [1]), it is customary to consider the case where the largest number of particles are interacting, namely when $K=0$ as shown in Fig. 1. In this approximation, one introduces the DOS for one spin $\varrho_{1}(\epsilon)$ in (10) and assumes that it is constant around the Fermi surface. This is strictly true in $2 \mathrm{D}$ and otherwise a good approximation in any D provided that $\hbar \omega_{D} / E_{F} \ll 1$. Then, the binding energy $\Delta_{0}$ for $K=0$ CPs turns out to be given by the familiar limit for the Cooper interaction [2]

$$
\Delta_{0} \underset{\lambda \rightarrow 0}{\longrightarrow} 2 \hbar \omega_{D} \exp (-2 / \lambda)
$$

where $\lambda \equiv \varrho_{1}\left(E_{F}\right) V_{0}$ is the usual dimensionless coupling parameter. Note that the CP $\Delta_{0}$ vanishes like $\exp (-2 / \lambda)$ unlike the BCS gap [1] which vanishes like $\exp (-1 / \lambda)$.

For a contact interaction of the form $-v_{0} \delta(x)$, where $x$ is the separation between the two fermions, the strength $v_{0}$ has dimensions of energy $\times$ length and the pair binding energy can again be calculated using the same approximation, namely taking the DOS at the Fermi level. After some manipulation the small-coupling limit of $\Delta_{0}$ is (see Ref. 42. and esp. Ref. [43] Eq. 17)

$$
\Delta_{0} \underset{\lambda \rightarrow 0}{\longrightarrow} 8 E_{F} \exp (-2 / \lambda)
$$

where in this case the dimensionless coupling parameter is given by $\lambda \equiv \varrho_{1}\left(E_{F}\right) v_{0} / L$. This same result also follows from the Cooper interaction result in (16) on taking the limit $k_{D} \rightarrow \infty$. 


\section{CP dispersion relation}

We analyze the case of a contact interaction where the solutions are given in (14)-(16). As mentioned, the analytical solutions depend on the strength of the dimensionless coupling $\lambda$ and on the magnitude of $K$. When $K>2$ one must take into account an additional restriction due to the integration regions defined in (13) since for stronger coupling one would expect larger values for $\Delta_{K}$. Therefore, for a particular $\lambda$ we define the value $K_{c}(\lambda)$ for which $\left.\alpha_{K}\right|_{K=K_{c}}=0$ implying from (15) that $K_{c}^{2} / 4 \equiv 1-\Delta_{K_{c}} / 2$ where $\Delta_{K_{c}}$ is the CP energy for $K=K_{c}$. The transcendental equations for the CP energy $-\Delta_{K}$ are then, again if $\beta_{K}^{2} \equiv-\alpha_{K}^{2}$,

$$
\frac{1}{\lambda}=\left\{\begin{array}{lll}
-\frac{1}{2 \alpha_{K}} \ln \left[\left(1+K / 2-\alpha_{K}\right) /\left(1+K / 2+\alpha_{K}\right)\right] & \text { for } K<2, \quad 0<\lambda<2 & \text { a) } \\
\frac{1}{\beta_{K}}\left[\pi / 2-\tan ^{-1}\left([1+K / 2] / \beta_{K}\right)\right] & \text { for } K<2, \quad 2<\lambda<\infty & \text { b) } \\
-\frac{1}{2 \alpha_{K}} \ln \left[\left(1+K / 2-\alpha_{K}\right) /\left(1+K / 2+\alpha_{K}\right)\right] & \text { for } K_{c}<K<2, \quad 1<\lambda<2 & \text { c) } \\
\frac{1}{\beta_{K}}\left(\pi / 2-\tan ^{-1}\left[(1+K / 2) / \beta_{K}\right]\right) & \text { for } 0<K<K_{c}, \quad 1<\lambda<2 & \text { d) } \\
\frac{1}{\beta_{K}}\left(\pi / 2-\tan ^{-1}\left[(K / 2+1) / \beta_{K}\right]+\tan ^{-1}\left[(K / 2-1) / \beta_{K}\right]\right) & \text { for } K>2, \lambda>0 . & \text { e) }
\end{array}\right.
$$

The existence of analytically different solutions follows from the graphical construct shown in Fig. 2 where we plot the rhs of (19) and $1 / \lambda$ as a function of the possible values of the CP energy $\Delta_{K}$ for different values of $K$. For convenience, we label as type-I those solutions that satisfy $(19 \mathrm{a})$ and $(19 \mathrm{k})$; the other solutions are labeled type-II. Solutions $\Delta_{K}$ only exist at those points where the $K$-curves cross the $1 / \lambda$ lines. From this construct three regions can be distinguished:

a) Weak coupling $0<\lambda<1$ and $\left(\Delta_{K} / 2+K^{2} / 4-1\right)<0$. In this region, there will always be a type-I solution for small values of $K$.

b) Intermediate coupling $1<\lambda<2$ where $\alpha_{K}^{2}$ changes sign and therefore both types of solution are present.

c) Strong coupling $\lambda>2$ and $\left(\Delta_{K} / 2+K^{2} / 4-1\right)>0$. In this region, only type-II solutions are possible.

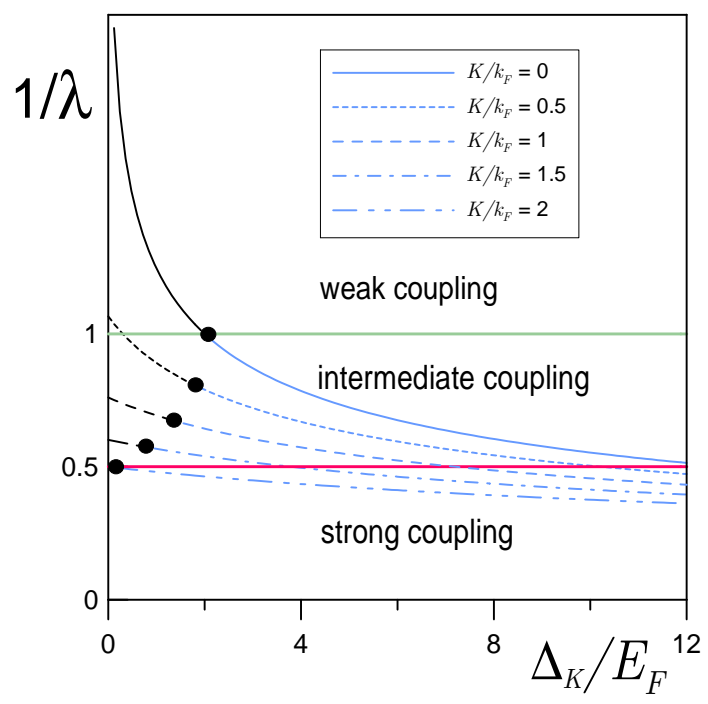

Figure 2: Graphical determination of CP energies $\Delta_{K}$ for different strengths $\lambda$ in (19). Horizontal lines are lhs and curves are rhs of (19) for five different values of $K$. The solution $\Delta_{K}$ is given by the abscissa where the corresponding $K$-curve crosses (dots) the $1 / \lambda$ line. Note that there are some intervals of $\lambda$ and of $K$ without solutions. Black dots on curves associated with a fixed $K$ define points at which the analytic forms on rhs of (19) change from one to another.

For $K=0(19 \mathrm{a})$ reduces to

$$
\exp \left(-\frac{2}{\lambda} \sqrt{1-\Delta_{0} / 2}\right)=\frac{1-\sqrt{1-\Delta_{0} / 2}}{1+\sqrt{1-\Delta_{0} / 2}}
$$




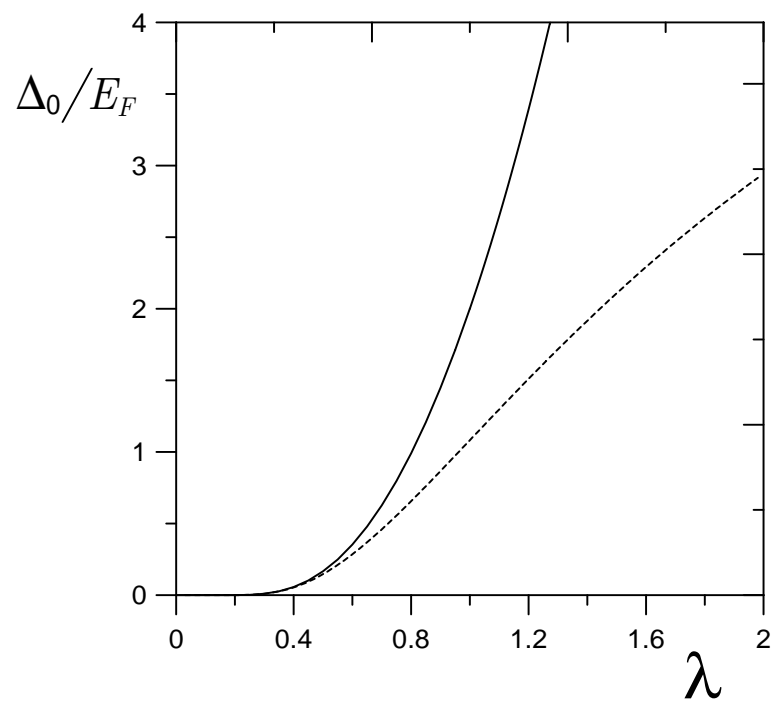

Figure 3: Exact $K=0 \mathrm{CP}$ energy $\Delta_{0}$ for the contact interaction as function of coupling $\lambda$ (full curve) as obtained from (20) compared to the weak-coupling approximation (18) (dashed curve).

As a consistency check we determine the CP energy $\Delta_{K}$ when the Fermi sea vanishes, i.e., $E_{F} \rightarrow 0$ and $k_{F} \rightarrow 0$ or when the $\mathrm{CP}$ is in vacuo. We note that only (19) is meaningful in this limit since $\tilde{K}<2$ implies $K<2 k_{F}$ in wavenumber units so that $K \leq 0$ when $k_{F} \rightarrow 0$ which contradicts the assumption made just below (13) that $K>0$. Introducing in (19) ) the value of $\lambda$ defined in (11) in terms of $k_{F}$ and reverting to explicit energy and wavenumber units one obtains

$$
\frac{\beta_{K}}{\lambda}=\pi / 2-\tan ^{-1}\left[\left(K / 2 k_{F}+1\right) / \beta_{K}\right]+\tan ^{-1}\left[\left(K / 2 k_{F}-1\right) / \beta_{K}\right]
$$

or

$$
\begin{aligned}
\frac{\sqrt{\Delta_{K} m / \hbar^{2}+K^{2} / 4-k_{F}^{2}}}{m V_{0} / \pi \hbar^{2}}=\pi / 2 & -\tan ^{-1}\left[\left(K / 2+k_{F}\right) / \sqrt{\Delta_{K} m / \hbar^{2}+K^{2} / 4-k_{F}^{2}}\right] \\
& +\tan ^{-1}\left[\left(K / 2-k_{F}\right) / \sqrt{\Delta_{K} m / \hbar^{2}+K^{2} / 4-k_{F}^{2}}\right] .
\end{aligned}
$$

When $k_{F} \rightarrow 0$ this leads to

$$
-\Delta_{K}=-\frac{m V_{0}^{2}}{4 \hbar^{2}}+\frac{\hbar^{2} K^{2}}{4 m}
$$

which is the expected actual energy of a composite object of mass $2 m$, self-bound via a 1D delta potential of arbitrary strength $V_{0}$, with its single-bound-state binding energy [45, 46] $m V_{0}^{2} / 4 \hbar^{2}$ and moving freely in vacuo.

When $K \ll 1$ we may assume the series expansion $\Delta_{K} \simeq \Delta_{0}+\Delta_{1} K+\Delta_{2} K^{2}+\cdots$ in (20). For weak coupling, $\lambda \rightarrow 0$ implies that $\Delta_{0} \longrightarrow 0$ so that $\sqrt{1-\Delta_{0} / 2} \simeq 1-\Delta_{0} / 4+\cdots$ which leads to (18) as expected. The same result is obtained using the DOS approximation given in (17) above. In Fig. 3 we show the exact result for $\Delta_{0}$ compared to (18) for weak coupling. The coefficient of the linear term $\Delta_{1}$ is of special interest. It can be obtained explicitly if we assume weak coupling and using the fact that $\Delta_{0}$ can be neglected in the exponentials, that is, by assuming that $\exp \left(-2 \alpha_{K} / \lambda\right) \simeq \exp (-2 / \lambda)$. The result is

$$
\Delta_{1} \simeq-2 \tanh ^{2}(1 / \lambda)
$$

A similar procedure can be used to obtain the second-order coefficient

$$
\Delta_{2} \simeq-\tanh ^{4}(1 / \lambda) .
$$




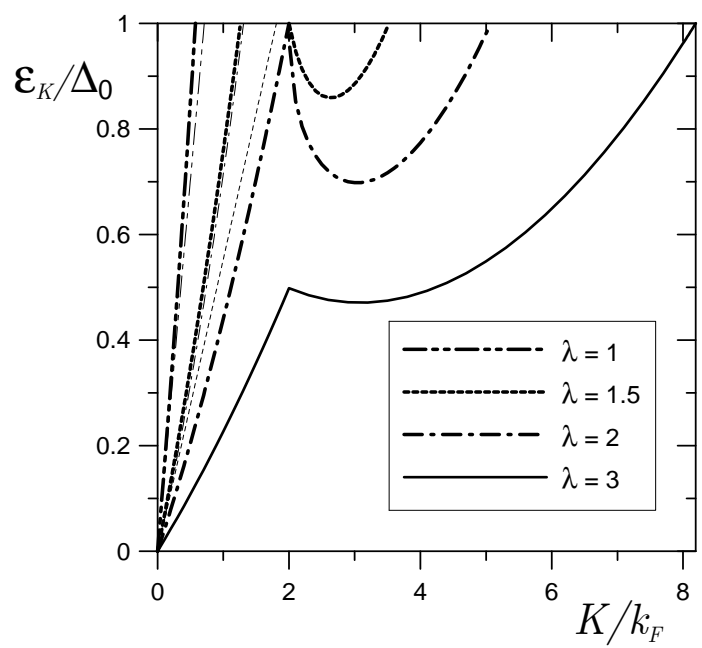

Figure 4: Pair excitation energy $\mathcal{E}_{K} \equiv \Delta_{0}-\Delta_{K}$ in units of $\Delta_{0}$ for a contact interaction for different values of $\lambda$ (thick curves). Thin curves refer to pure linear while thin dot-dashed curve for $\lambda=1.5$ is the linear plus quadratic behavior. For weak-coupling $(\lambda \leq 1)$, only the linear phonon-like term is present with the pair breaking up whenever $\mathcal{E}_{K} \geq 1$. For larger coupling, e.g., $\lambda \geq 1.5$, a roton-like branch appears when $\tilde{K} \geq 2$.

Therefore, the dispersion relation for $\Delta_{K}$ up to second order in $K$ but for weak coupling $\lambda$ in explicit energy and wavenumber units is

$$
\begin{aligned}
& \Delta_{K} \simeq \Delta_{0}-\tanh ^{2}(1 / \lambda) \hbar v_{F} K-\tanh ^{4}(1 / \lambda) \frac{\hbar^{2} K^{2}}{2 m}+\cdots \\
& \underset{\lambda \rightarrow 0}{\longrightarrow} 8 E_{F} \exp (-2 / \lambda)-\hbar v_{F} K-\frac{\hbar^{2} K^{2}}{2 m}+\cdots
\end{aligned}
$$

where (18) was used. The negative signs in the first- and second-order terms for a given coupling $\lambda$ implies that the pair will break-up as $K$ increases beyond a certain value for which $\Delta_{K}=0$. However, for $K \geq 2 k_{F}$ and for sufficiently large $\lambda$, a pair can become bound again as shown in Fig. 4 where we plot the gapped excitation energy $\mathcal{E}_{K} \equiv \Delta_{0}-\Delta_{K}$ as a function of $K$ for different couplings. The special case of $\lambda=1.5$ illustrates this behavior. As $K$ increases from zero, the excitation energy is essentially linear up to $K \simeq 1.2 k_{F}$ when the pair breaks up but for $K \geq 2 k_{F}$ the pair comes back into existence with an excitation energy that is close to quadratic in the pair wavenumber $K$.

\section{Finite-range interactions}

In contrast with the cases mentioned above, here we consider a more general interaction between fermions where a range parameter is introduced albeit the interaction form is still separable. In $3 \mathrm{D}$ it is customary to introduce a screened interaction of the Yukawa form $\exp \left(-r / r_{0}\right) / r$ which in momentum space is $\propto\left[\left(q^{2}+\left(1 / r_{0}\right)^{2}\right]^{-2}\right.$, where $q$ is the momentum transfer wavenumber. Based on this criterion and following previous calculations (cf. Ref. [44] esp. Eq. 10) we write the form factor $g(k)$ in (4) as

$$
g(k)=\frac{\theta(|k|-[1+K / 2])}{\sqrt{k^{2}+k_{0}^{2}}}
$$

where all wavenumbers are again in units of $k_{F}$. The characteristic equation for the energy (10) becomes

$$
\frac{1}{\lambda}=\int_{1+K / 2}^{\infty} \frac{d k}{\left(k^{2}+\Delta_{K} / 2+K^{2} / 4-1\right)\left(k^{2}+k_{0}^{2}\right)} .
$$


This equation can be solved exactly and leads to the transcendental equations for the CP energy

$$
\begin{array}{rlrl}
\frac{\left(k_{0}^{2}+\alpha_{K}^{2}\right)}{\lambda} & =-\frac{1}{2 \alpha_{K}} \ln \left[\frac{1+K / 2-\alpha_{K}}{1+K / 2+\alpha_{K}}\right]-\frac{\pi}{2 k_{0}}+\frac{1}{k_{0}} \tan ^{-1}\left[\frac{1+K / 2}{k_{0}}\right] & K / 2 \ll 1, \quad \alpha_{K}^{2}>0 \\
\frac{k_{0}^{2}-\beta_{K}^{2}}{\lambda}=\frac{1}{\beta_{K}}\left(\frac{\pi}{2}-\tan ^{-1}\left[\frac{1+K / 2}{\beta_{K}}\right]\right)-\frac{1}{k_{0}}\left(\frac{\pi}{2}-\tan ^{-1}\left[\frac{1+K / 2}{k_{0}}\right]\right) & K / 2<1, \alpha_{K}^{2}<0 \\
\frac{k_{0}^{2}-\beta_{K}^{2}}{\lambda}=\frac{1}{\beta_{K}}\left(\frac{\pi}{2}+\tan ^{-1}\left[\frac{K / 2-1}{\beta_{K}}\right]-\tan ^{-1}\left[\frac{1+K / 2}{\beta_{K}}\right]\right) & \\
-\frac{1}{k_{0}}\left(\frac{\pi}{2}+\tan ^{-1}\left[\frac{K / 2-1}{k_{0}}\right]-\tan ^{-1}\left[\frac{1+K / 2}{\beta_{K}}\right]\right) & K / 2>1, \quad \alpha_{K}^{2}<0
\end{array}
$$

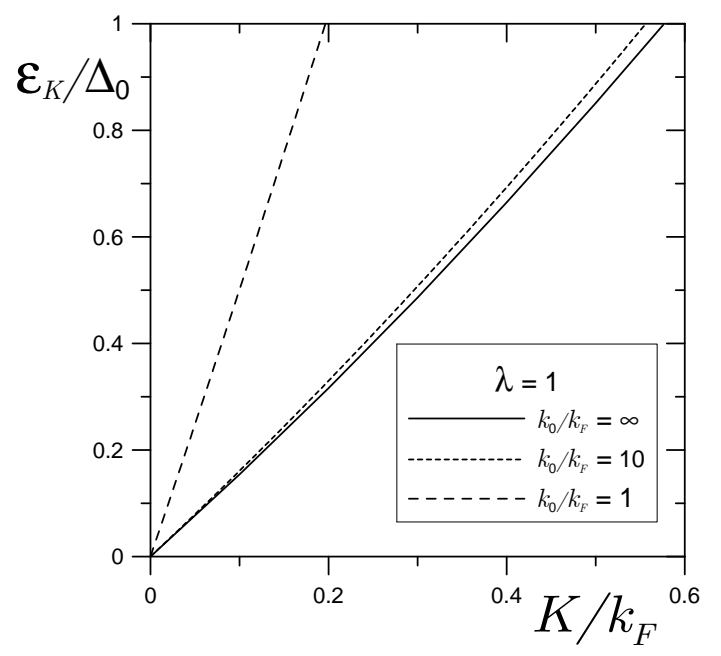

Figure 5: Excitation energy $\mathcal{E}_{K}$ in units of $\Delta_{0}$ of a CP for moderate coupling $\lambda=1$ and for different interaction ranges gauged by the inverse range parameter $k_{0}$. The dispersion relation is essentially linear over the entire range of $K$ below the pair-breaking limit $\mathcal{E}_{K} / \Delta_{0}=1$. Infinite $k_{0}$ is the contact-interaction limit.

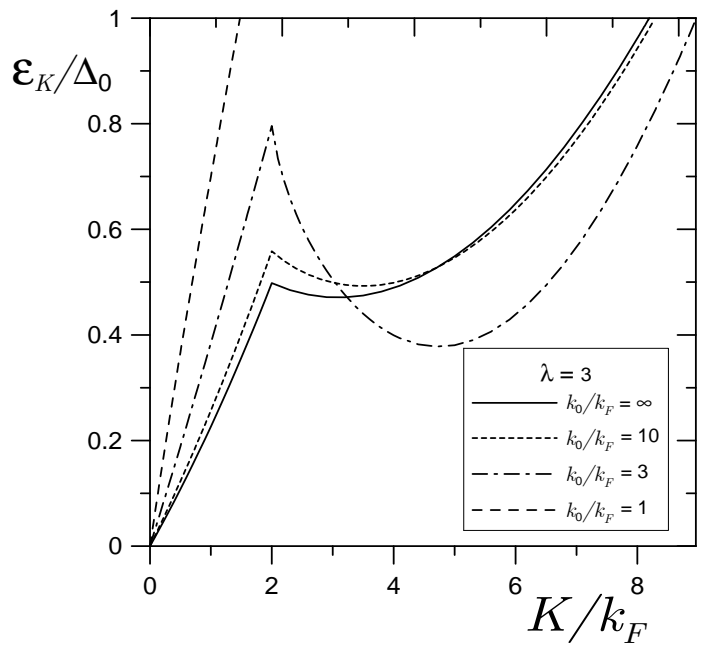

Figure 6: Excitation energy $\mathcal{E}_{K}$ in units of $\Delta_{0}$ of a CP for strong coupling, $\lambda=3$, for different values of inverse range parameter $k_{0}$. For $k_{0} \geq 3$ the two distinct dispersion branches are possible. Infinite $k_{0}$ refers to the contact-interaction limit.

Again, there are three regions for the existence of stable pairs. In Fig. 5 we plot the pair energy as a function of $K$ for weak coupling and for different values of the range parameter $1 / k_{0}$. Stronger coupling spectra are shown in Fig. 6] 
where we plot the binding energies for $\lambda=3$ where both, the linear and roton-like modes are present [47]. The sharp cusp separating phonon-like and roton-like modes at precisely $K=2 k_{F}$ is a unique characteristic of the $1 \mathrm{D}$ system. It appears to be a precursor of the smooth "maxon-like" hump in 2D (Ref. [40], Figs. 1 and 3) and less pronounced in 3D (Ref. [41], Fig. 2).

\section{Conclusions}

Notwithstanding the obvious simplicity of a model consisting of a many-fermion system where particles interact only in the vicinity of the Fermi level through two-body, attractive, separable interactions in the background of an ideal 1D Fermi gas, it reveals novel, unique properties, particularly those related to the energy of Cooper pairs moving with nonzero center-of-mass momentum (CMM) $K$. The fact that one can calculate exact expressions for the pair energy with different separable interactions allows us to construct the collective excitation spectrum of Cooper pairs for any coupling $\lambda$ and any value of $K$. For $K<k_{F}$, the excitation energy has a linear term in $K$. As the CMM wavenumber $K$ increases the pair eventually breaks up. However, for sufficiently strong coupling, there is an additional collective mode for $K \geq 2 k_{F}$ with a roton-like dispersion. For a contact interaction, the two modes are disconnected if the coupling is weak but for stronger coupling the excitation spectrum exhibits both modes. Introducing more realistic interactions that include screening effects shows a similar behavior.

The sharp cusp separating phonon-like and roton-like modes at precisely $K=2 k_{F}$ is a unique characteristic of the 1D system. It appears to be a precursor of the smooth "maxon-like" hump in 2D (Ref. [40], Figs. 1 and 3) and less pronounced in 3D (Ref. 41], Fig. 2). The smoothness could be due to angular integrations in 2D and 3D washing out the beaked transition between both modes found here in 1D.

Acknowledgments We acknowledge partial support from grants PAPIIT IN114708, IN106908 and CONACyT 104917.

\section{References}

[1] J. Bardeen, L.N. Cooper and J.R. Schrieffer, Phys. Rev. 108, 1175 (1957).

[2] L.N. Cooper, Phys. Rev. 104, 1189 (1956).

[3] J.R. Schrieffer, Theory of Superconductivity (Benjamin, Reading, MA, 1983).

[4] B.S. Deaver, Jr. and W.M. Fairbank, Phys. Rev. Lett. 7, 43 (1961).

[5] R. Doll and M. Näbauer, Phys. Rev. Lett. 7, 51 (1961).

[6] C.E. Gough, M.S Colclough, E.M. Forgan, R.G. Jordan, M. Keene, C.M. Muirhead, I.M. Rae, N. Thomas, J.S. Abell, and S. Sutton, Nature 326, 855 (1987).

[7] J.G. Bednorz and K.A. Müller, Z. Phys. 64, 189 (1986).

[8] M.K. Wu, J.R. Ashburn, C.J. Torng, P.H. Hor, R.L. Meng, L. Gao, Z.J. Huang, Y.Q. Wang, and C.W. Chu, Phys. Rev. Lett. 58, 908 (1987).

[9] L. Gao, Y.Y. Xue, F. Chen, Q. Xiong, R.L. Meng, D. Ramírez, C.W. Chu, J.H. Eggert, and H.K. Mao, Phys. Rev. B 50, 4260 (1994).

[10] C. Buzea and K. Robbie, Supercond. Sci. Technol. 18, R1 (2005).

[11] M.K. Wu, D.Y. Chen, F.Z. Chien, S.R. Sheen, D.C. Ling, C.Y. Tai, G.Y. Tseng, D.H. Chen, and F.C. Zhang, Z. f. Physik 102, 37 (1996).

[12] D.Y. Chen, F.Z. Chiena, D.C. Lingb, J.L. Tsengb, S.R. Sheenb, M.J. Wangb, and M.K. Wub, Physica C 282-287, 73 (1997). 
[13] H.A. Blackstead, et al., Cubic $\mathrm{Ba}_{2} Y R u_{1-x} C u_{x} \mathrm{O}_{6}$ : a possible strong-coupled SDW superconductor (unpublished).

[14] P.W. Anderson, The Theory of Superconductivity in the High $T_{c}$ Cuprates (Princeton University Press, Princeton, NJ, 1997).

[15] P.A. Lee, N. Nagaosa, and X.G. Wen, Rev. Mod. Phys. 78, 17 (2006).

[16] N. Bulut, Adv. Phys. 51, 1587 (2002).

[17] D. Manske, Theory of Unconventional Superconductors: Cooper Pairing Mediated by Spin Excitations (Springer Tracts in Modern Physics 202, Springer, Berlin, 2004)

[18] A.V. Chubukov, D. Pines, and J. Schmalian, in The Physics of Superconductors, Vol. 1: Conventional and High- $T_{c}$ Superconductors, pp. 495-590, K.H. Bennemann and J.B. Ketterson (eds.) (Springer, Berlin, 2003).

[19] R.B. Laughlin, cond-mat/0209269 B.A. Bernevig, R.B. Laughlin, and D.I. Santiago, Phys. Rev. Lett. 91, 147003 (2003); K. Maki, S. Haas, D. Parker, H. Won, B. Dora, and A. Virosztek, Phys. Stat. Sol. 3, 3156 (2006).

[20] J.F. Annett, N.D. Goldenfeld, and A.J. Leggett, in Physical Properties of High Temperature Superconductors V, pp. 375-461, D.M. Ginsberg (ed.) (World Scientific, Singapore, 1996).

[21] C.C. Tsuei and J.R. Kirtley, Rev. Mod. Phys. 72, 969 (2000).

[22] T. Maier, M. Jarrell, T. Pruschke, and J. Keller, Phys. Rev. Lett. 85, 1524 (2000).

[23] H. Ott, in The Physics of Superconductors, Vol. 1: Conventional and High $T_{c}$ Superconductors, pp. 385-481, K.H. Bennemann and J.B. Ketterson (eds.), (Springer, Berlin, 2003).

[24] R. Friedberg and T.D. Lee, Phys. Rev. B 40, 6745 (1989).

[25] R. Friedberg, T.D. Lee, and H.-C. Ren, Phys. Rev. B 42, 4122 (1990).

[26] R. Friedberg, T.D. Lee, and H.-C. Ren, Phys. Lett. A 152, 417 and 423 (1991).

[27] R. Friedberg, T.D. Lee, and H.-C. Ren, Phys. Rev. B 45, 10732 (1992).

[28] J. Ranninger and S. Robaszkiewicz, Physica B 135, 468 (1985).

[29] J. Ranninger, R. Micnas, and S. Robaszkiewicz, Ann. Phys. Fr. 13, 455 (1988).

[30] A.S. Alexandrov and N.F. Mott, Rep. Progs. Phys. 57, 1197 (1994).

[31] D.M. Eagles, Phys. Rev. 186, 456 (1969).

[32] R. Micnas, J. Ranninger, and S. Robaszkiewicz, Rev. Mod. Phys. 62, 113 (1990).

[33] R. Micnas, S. Robaszkiewicz, and A. Bussmann-Holder, Struct. Bond 114, 13 (2005).

[34] Q. Chen, J. Stajic, Sh. Tan, and K. Levin, Phys. Repts. 412, 1 (2005).

[35] S.K. Adhikari, M. de Llano, F.J. Sevilla, M.A. Solís, and J.J. Valencia, Physica C 453, 37 (2007).

[36] M. Botti, E. Cappelluti, C. Grimaldi, and L. Pietronero, Phys. Rev. B 66, 054532 (2002).

[37] V.V. Tolmachev, Phys. Lett. A 266, 400 (2000).

[38] M. de Llano and V.V. Tolmachev, Physica A 317, 546 (2003)

[39] M. de Llano and V.V. Tolmachev, Ukranian J. Phys. 55, 79 (2010).

[40] S. K. Adhikari, M. Casas, A. Puente, A. Rigo, M. Fortes, M. A. Solís, M. de Llano, A. A. Valladares, and O. Rojo, Phys. Rev. B 62, 8671 (2000). 
[41] S. K. Adhikari, M. Casas, A. Puente, A. Rigo, M. Fortes, M. A. Solís, M. de Llano, A. A. Valladares, and O. Rojo, Physica C 351, 341 (2001).

[42] M. Casas, C. Esebbag, A. Extremera, J. M. Getino, M. de Llano, A. Plastino, and H. Rubio, Phys. Rev. A 44, 4915 (1991).

[43] V.C. Aguilera-Navarro and M. de Llano, Rev. Mex. Fís. 40, 167-173 (1994).

[44] P. Nozières and S. Schmitt-Rink, J. Low Temp. Phys. 59, 195 (1985).

[45] S. Gasiorowicz, Quantum Mechanics (Wiley, NY, 1974) p. 93.

[46] M. de Llano, Mecánica Cuántica (Las Prensas de Ciencias, UNAM, México, DF, 2002) in Spanish, p. 46.

[47] R.J. Donnelly, Phys. Today, p. 34 (October, 2009) 